\title{
El presupuesto por programas en la administración local española
}

\author{
Juan Calvo Vérgez \\ Profesor Titular de Derecho Financiero y Tributario \\ Universidad de Extremadura
}

Sumario:_I. UNA CONSIDERACIÓN PRELIMINAR: EL MARCO PRESUPUESTARIO DE LAS HACIENDAS LOCALES. - II. CONFIGURACIÓN DEL PRESUPUESTO POR PROGRAMAS: CONCEPTO Y OBJETIVOS.-III. PRINCIPIOS INFORMADORES DEL PRESUPUESTO POR PROGRAMAS. - IV. PRINCIPALES FASES DEL PRESUPUESTO POR PROGRAMAS. V. LA PROYECCIÓN DEL PRESUPUESTO POR PROGRAMAS EN EL MARCO DE LAS HACIENDAS LOCALES. 1. Introducción. 2. Criterios clasificatorios de los presupuestos de los entes locales.

Resumen: El presente trabajo tiene por objeto analizar la aplicación de la técnica presupuestaria de los presupuestos por programas en el ámbito municipal. Tras una delimitación inicial de la especial significación que adquieren los presupuestos generales de las Haciendas Locales, se estudia la proyección sobre dichos presupuestos de diversas cuestiones relativas a la presupuestación por programas tales como los objetivos perseguidos, las fases que han de seguirse en su elaboración, los principios que deben ser respetados o los criterios clasificatorios que han de aplicarse.

Palabras clave: Presupuestos, programas, objetivos, Municipios, clasificación, evaluación, control.

\section{UNA CONSIDERACIÓN PRELIMINAR: EL MARCO PRESUPUESTARIO DE LAS HACIENDAS LOCALES}

Como es sabido, desde un punto de vista económico el Presupuesto representa un plan de la actividad financiera y, por tanto, una ordenación de las necesidades públicas de acuerdo con su respectiva prioridad. $Y$ es que, constituyendo aquél una previsión normativa que establece las pautas para la gestión económica de los Entes públicos y la asignación de recursos financieros de los que dispone el Sector público, nos hallamos ante una pieza fundamental en la Administración Pública y, más precisamente, en la gestión pública. 
Dos son los principales elementos a través de los cuales resulta posible conceptuar el Presupuesto. De una parte, el contenido mínimo que ha de incluir, esto es, el conjunto de obligaciones y derechos presentes en el mismo o, dicho de otro modo, los ingresos y gastos. De otra, los efectos jurídicos que produce, es decir, los gastos que como máximo se pueden efectuar, así como la estimación de los ingresos que se van a conseguir durante el ejercicio presupuestario. En definitiva, la ordenación jurídica del gasto público, en sus aspectos financieros (asignación de recursos, ejecución del proceso de gasto y control del mismo) se desarrolla en torno al Presupuesto.

El Presupuesto representa por tanto el plan de gastos y previsión de ingresos del Estado o de los restantes entes públicos territoriales (en nuestro caso, de los Entes Locales) para un determinado ejercicio económico, constituyendo un instrumento de racionalización y organización de la actividad financiera y, en general, de la actuación económica del Sector Público.

Por otra parte, el Presupuesto se presenta como una norma atributiva de competencia. $Y$ es que al autorizar los gastos, con su correspondiente límite y destino, el legislador está permitiendo a la Administración alcanzar diversas finalidades, pudiendo disponer al efecto de los fondos previstos para ellas.

Es precisamente esta autorización de gastos la que refleja los efectos jurídicos del Presupuesto, calificándose como «crédito presupuestario» cada una de las cifras consignadas en los mismos que se autoriza a la Administración a gastar. Estas cifras encierran además un triple límite. En primer lugar, de carácter cualitativo dado que, de conformidad con lo establecido en el art. 42 de la Ley 47/2003, de 26 de noviembre, General Presupuestaria (LGP), los créditos para gastar «se destinarán exclusivamente a la finalidad específica para la que hayan sido autorizados por la Ley de Presupuestos o a la que resulte de las modificaciones aprobadas conforme a esta Ley».

En segundo término cabe aludir a la existencia de un límite cuantitativo, en virtud del cual se prohíbe a la Administración adquirir compromisos de gasto «por cuantía superior al importe de los créditos autorizados» (art. 46 LGP). Finalmente cabe destacar la existencia de un límite temporal, contemplado en el art. 49.1 LGP, y en virtud del cual se ordena que con cargo a los créditos de cada presupuesto sólo puedan contraerse obligaciones derivadas de adquisiciones, obras, servicios y demás prestaciones o gastos realizados, en general, en el propio ejercicio presupuestario. En armonía con todos estos límites establece además el art. 49.2 LGP que aquellos créditos para gastos que no se hubiesen utilizado el último día del ejercicio quedarán anulados de pleno derecho, salvo ciertas excepciones previstas en la propia Ley.

Pues bien, al igual que sucede con otros entes territoriales tales como las Comunidades Autónomas (CC.AA.), también las Corporaciones Locales ven reflejados en su régimen presupuestario los pilares básicos que asientan su autonomía en materia tributaria. En efecto, la autonomía financiera de Ayuntamientos y Diputaciones, cons80 titucionalmente reconocida, implica necesariamente la autonomía presupuestaria 
sobre unas bases similares a las existentes en la esfera estatal'. Ahora bien, debido a la falta de potestad legislativa que, como es sobradamente conocido, caracteriza al ámbito local, la regulación general de la institución presupuestaria queda contenida en una norma de carácter estatal, el Real Decreto Legislativo 2/2004, de 5 de marzo, por el que se aprueba el Texto Refundido de la Ley Reguladora de las Haciendas Locales (TRLRHL), a cuyos preceptos debe atenerse cada Corporación Local en su elaboración, aprobación y ejecución.

Define concretamente el art.162 del citado Texto Refundido los Presupuestos Generales de las Entidades Locales como «La expresión cifrada, conjunta y sistemática de las obligaciones que, como máximo, pueden reconocer la entidad, y sus organismos autónomos, y de los derechos que prevean liquidar durante el correspondiente ejercicio, así como de las previsiones de ingresos y gastos de las sociedades mercantiles cuyo capital social pertenezca íntegramente a la entidad local correspondiente»².

Nótese por tanto que el Presupuesto constituye el documento que rige la vida económica de la Administración Local y, por tanto, el instrumento principal y vertebrador de su gestión política, sobre el que se articula, a lo largo de todo el año, el conjunto de su organización, previéndose los ingresos a liquidar, estimándose y limitándose los gastos a ejecutar, autorregulándose la actividad económica, financiera, etc. Y todo ello en relación, no ya sólo con el Presupuesto de la propia Entidad, sino también con el de sus Organismos autónomos y sociedades de capital íntegramente local. Piénsese que todo acto de una Entidad local, en su conjunto, con trascendencia económica y realizado durante el ejercicio económico (coincidente con el año natural), además de emanar del órgano administrativo competente, debe tener el respal-

1 Véanse a este respecto, y con carácter general, Palao Tabodada, C. (1971), «Los Presupuestos de los Entes Locales», Hacienda Pública Española, núm. 11, 1971, pp.. 47 y ss. y (1987) «Los presupuestos de las entidades locales», en la obra colectiva Manual de Derecho Tributario Local, Escola d'Administració Publica de la Generalitat de Catalunya, pp.. 133 y ss., Lozano Serrano, C., (1982) «La autonomía municipal en materia de gastos: tendencias del ordenamiento", Presupuesto y Gasto Público, núm. 12, pp.. 34 y ss., Soler Roch, M. ${ }^{a}$ T., (1990) «El Régimen Presupuestario de las Entidades Locales: un análisis comparativo con el régimen presupuestario estatal», Palau 14-Revista Valenciana de Hacienda Pública, núm. 12, pp.. 66 y ss., Checa González, C., (1990) Los presupuestos generales de las Entidades Locales, Lex Nova, Valladolid, pp. 23 y ss., Arnau Bernia, V. (1990), «El régimen presupuestario de las Entidades Locales», Revista de Hacienda Autonómica y Local, núm. 58, 1990, pp. 54 y ss. y (1991) Manual de Presupuestos de las Entidades Locales, Marcial Pons,, pp. 34 y 35, Arnal Suría, S. y González Pueyo, J. M., (1995) Manual de Presupuestos y Contabilidad de las Corporaciones Locales, pp. 38 y ss., Alarcón García, G., (1996) El Presupuesto General de los Municipios, Tecnos, Madrid, pp.. 72 y 73 y Valenzuela Villarubia, I., (2007) El régimen Presupuestario de los Entes Locales. El Presupuesto como instrumento de gestión y planificación, Thomson-Aranzadi, Pamplona, pp. 51 y ss.

2 Ya con anterioridad la antigua Ley 39/1988, de 28 de diciembre, reguladora de las Haciendas Locales, definía los Presupuestos Generales de estas Entidades del mismo modo que lo hacía el extinto Texto Refundido de la Ley General Presupuestaria (TRLGP) al conceptuar los Presupuestos estatales, disponiéndose en su art. 143 (e igualmente en el art. 2 del antiguo Reglamento Presupuestario) que «Los Presupuestos Generales de las Entidades Locales constituyen la expresión cifrada conjunta y sistemática de las obligaciones que, como máximo, pueden reconocer la Entidad, y sus Organismos Autónomos, y de los derechos que prevean liquidar durante el correspondiente ejercicio, así como de las previsiones de ingresos y gastos de las Sociedades Mercantiles cuyo capital social pertenezca íntegramente a la Entidad local correspondiente». 
do de una consignación presupuestaria, ajustándose a los procedimientos establecidos para la ejecución del presupuesto. Adicionalmente deberá tener un reflejo contable y ser fiscalizado. Y ello referido, no ya sólo a la organización centralizada, sino también a los órganos dotados de personalidad jurídica pública o privada.

Varias son las características configuradoras del actual marco legal presupuestario local. En primer lugar, se impone la elaboración de un Presupuesto único. Y, en este sentido, los Presupuestos Generales de la Entidad Local constituyen, como ya se ha indicado, la expresión cifrada, conjunta y sistemática de las obligaciones que, como máximo (carácter limitativo) pueden reconocer la Entidad y sus Organismos autónomos, así como de los derechos que prevean liquidar (carácter estimativo) durante el correspondiente ejercicio y de las previsiones de ingresos y gastos de las Sociedades Mercantiles cuyo capital social pertenezca íntegramente a la Entidad local correspondiente.

La definición que de los Presupuestos Generales de las Entidades Locales nos ofrece el RDLeg. 2/2004 permite realizar una serie de consideraciones preliminares. Nótese, en primer lugar, que se habla de «Presupuestos generales» y no de «Presupuesto». Y ello debido a que se trata, en realidad, de varios presupuestos: el de la propia Entidad, el de sus Organismos autónomos, y los estados de previsión de las sociedades mercantiles cuyo capital pertenezca íntegramente a la Entidad local. Los Presupuestos Generales integran así el conjunto del Sector público en un único presupuesto a través del llamado «estado de consolidación». Ahora bien materialmente sigue existiendo una pluralidad de ellos, cada uno de los cuales tiene su propia ejecución separada. Adicionalmente cada uno de los presupuestos que se integran en el Presupuesto General debe aprobarse sin déficit inicial, no pudiendo tampoco presentar déficit a lo largo del ejercicio, en aras de garantizar el necesario respeto al principio de estabilidad presupuestaria.

Cuando se afirma que dichos Presupuestos "constituyen la expresión cifrada (... )» se está aludiendo a la necesidad de que los derechos y obligaciones sean consignados numéricamente, lo que significa que los mismos deben ser valorados económicamente, siendo el resultado de dicha valoración lo que deberá reflejarse en el documento presupuestario. La trascripción del Presupuesto se efectúa así través de un documento de carácter numérico, posibilitándose de este modo el control sobre las autorizaciones recogidas en el presupuesto a partir de su contabilidad. Dichos derechos y obligaciones han de ser presentados además de un modo conjunto en el Presupuesto, quedando las cifras y datos ordenadas con arreglo a una determinada estructura. A partir de esta sistematización podrá llevarse a cabo una correcta consolidación de todos los documentos presupuestarios, pudiendo ser programada y planificada la actividad económico-financiera de los Entes Locales.

La utilización de la expresión «conjunta y sistemática» ha de ser interpretada en el sentido de que el Presupuesto de las Corporaciones Locales se expresa en un esta82 do de ingresos y en un estado de gastos. Tal y como precisaremos a continuación es- 
tos últimos, los gastos, presentan un carácter limitativo, no pudiendo adquirirse compromisos de gastos por cuantía superior a su importe. $Y$, por lo que a los ingresos se refiere, los mismos representan una mera previsión.

Dado que los derechos y obligaciones han de ser presentados de un modo conjunto, ambas partidas deben exhibirse simultáneamente en el Presupuesto, además de hacer alusión a la vigencia del principio de unidad presupuestaria. En efecto, la expresión sistemática de los derechos y obligaciones significa que las cifras y datos que lo constituyen han de ordenarse con arreglo a un sistema (orgánico, económico, funcional y por programas). A partir de esta sistematización podrá llevarse a cabo una correcta consolidación de todos los documentos presupuestarios, pudiendo ser programada y planificada la actividad financiera de los entes municipales.

¿Y cómo debe interpretarse la expresión «de las obligaciones que, como máximo, pueden reconocer la Entidad y sus Organismos autónomos»? En relación a los gastos el Presupuesto constituye una autorización para su realización y un límite en sentido cuantitativo, cualitativo y temporal (principio de especialidad). Por su parte, a través de la incorporación de la frase «y de los derechos que prevean liquidar durante el correspondiente ejercicio» se está señalando que, respecto de los ingresos, el Presupuesto constituye una previsión. Esta tiene una gran relevancia en el documento, ya que sobre la base de la misma se planifican los gastos que puedan ser realizados en el ejercicio presupuestario. Los ingresos señalados en el Presupuesto son fundamento y justificación de los gastos, de ahí que la Ley disponga la obligación de presentar, junto con el Presupuesto de la Entidad municipal, un informe económico-financiero.

El estado de ingresos del Presupuesto tiene efectos jurídicos, aunque éstos no sean de igual trascendencia que los derivados de gastos, ya que el Presupuesto no constituye ni un límite a la liquidación de derechos ni una autorización para la percepción de ingresos, al ser las Leyes reguladoras de cada uno de los derechos económicos las que disponen su gestión y recaudación, así como su vigencia, con independencia de las cantidades presupuestadas.

Nótese además que, al incluirse la afirmación «así como de las previsiones de ingresos y gastos de las Sociedades Mercantiles cuyo capital social pertenezca íntegramente a la Entidad local correspondiente», se está dando a entender que en relación con las sociedades mercantiles debe hablarse de «estados de previsión de ingresos y gastos». Estas previsiones son, con carácter general, y salvo que el Pleno disponga un régimen especial, meramente estimativas, por lo que carecen de relevancia jurídica y no son ni limitativas ni vinculantes.

Con carácter general las Entidades Locales deben elaborar y aprobar anualmente un presupuesto general que integre: el presupuesto de la propia Entidad, así como el de los Organismos autónomos dependientes de la misma; y los estados de previsión de ingresos y gastos de las sociedades mercantiles cuyo capital social pertenezca íntegramente a la Entidad Local. 
Como no puede ser de otra manera el ejercicio presupuestario coincide con el año natural, imputándose a él los derechos liquidados en el mismo, cualquiera que sea el periodo de que se deriven y las obligaciones reconocidas durante su vigencia, de acuerdo con las previsiones contenidas en el art.176 del TRLRHL.

Debe quedar claro pues desde un primer momento que las Corporaciones Locales disponen de una competencia exclusiva para decidir sobre la cuantía y el destino del gasto público local, elemento este definidor, como ya sabemos, de la autonomía financiera, más si cabe que las potestades tributarias. Por otra parte, la referida carencia de potestad legislativa determina el rango y la naturaleza meramente reglamentaria de sus Presupuestos, resultando así restringidas las posibilidades de creación e innovación del ordenamiento. Y dada la reserva de ley existente para establecer los modos de contraer obligaciones y realizar gastos por las Administraciones Públicas a que se refiere el art. 133.4 de la Constitución (CE), la regulación de estos aspectos centrales del Derecho Presupuestario ha de adquirir un carácter heterónomo, debiendo quedar fijado el régimen jurídico del Presupuesto Local, esto es, la normativa a la que habrán de sujetarse las Corporaciones Locales al aprobar y ejecutar su Presupuesto, por los entes superiores.

Dos son las Leyes estatales que, en la actualidad, delimitan la institución presupuestaria local. De una parte la Ley 7/1985, de 2 de abril, de Bases de Régimen Local (LBRL) y, más precisamente, su Título VIII. Y, de otra, el anteriormente citado Texto Refundido de la Ley Reguladora de las Haciendas Locales, cuyo Título VI se encarga de regular el Presupuesto y Gasto Público de los Entes Locales. Todo ello sin perjuicio de las especialidades que incorpora la Ley 57/2003, de 16 de diciembre, de Medidas para la modernización del Gobierno Local en sus arts. 133 y 136.

Cabe precisar no obstante que se mantienen en vigor las normas de desarrollo dictadas bajo la vigencia de la antigua Ley 39/1988, de 28 de diciembre, reguladora de las Haciendas Locales (LRHL), esto es, el Real Decreto 500/1990, de 20 de abril, por el que se desarrolló el Capítulo Primero del Título Sexto de la Ley 39/1988 en materia de presupuestos, y a través del cual se promulgó el denominado Reglamento Presupuestario de las Corporaciones Locales ${ }^{3}$, así como diversas disposiciones de menor rango destinadas a la regulación de cuestiones de carácter más concreto, caso por ejemplo de la Orden Ministerial de 8 de septiembre de 1999, que establece la estructura presupuestaria de las Entidades Locales.

Históricamente el proceso de acercamiento de la normativa presupuestaria de las Entidades Locales a los preceptos de la Ley General Presupuestaria se inició en 1979 con el Real Decreto Ley 11/1979 de 20 de julio, sobre Medidas Urgentes de Financiación de las Corporaciones Locales. A pesar de ello hasta la Ley 7/1985, de 2 de abril,

3 Este Real Decreto 500/1990, de 20 de abril, desarrolló los preceptos de la Ley de 1988 en relación a esta materia. Se trata de una norma que, si bien no fue adaptada al TRLHL, puede ser utilizada respecto a los artículos de la ley no modificados. 
Reguladora de las Bases de Régimen Local, las Entidades Locales permanecieron al margen del movimiento de modernización y normalización contable. Esta Ley se encargó de regular las líneas generales de un nuevo modelo de Hacienda Local deducido de las previsiones constitucionales.

Fue, por tanto, a raíz de la aprobación de la Ley 7/1985, de 2 de abril, posteriormente modificada en parte por la Ley 57/2003, de 16 de diciembre, de Medidas para la Modernización del Gobierno Local (LMMGL), cuando se inicia el período necesario para la adopción de una nueva normativa presupuestaria y contable en el ámbito de las Entidades locales. Dicho período tuvo su continuación en el ámbito presupuestario con la aparición de la Ley 39/1988 y de la Orden Ministerial de 20 de septiembre de 1989, reguladora de la actual estructura de los presupuestos de las Entidades Locales ${ }^{4}$.

En efecto la citada Ley 39/1988 diseñó las líneas básicas a las que debía adaptarse la contabilidad de las Entidades Locales y de sus Organismos autónomos atribuyendo al Ministerio de Hacienda, a propuesta de la Intervención General de la Administración del Estado, su desarrollo normativo completo. El plazo de implantación de la nueva normativa, que debía afectar a ejercicios completos, alcanzaba como máximo al ejercicio iniciado el 1 de enero de 1992, momento a partir del cual resultó de aplicación general.

No obstante, la implantación del nuevo sistema tributario local creado por esta Ley obligó a las Entidades Locales a servirse, desde 1 de enero de 1990, de una estructura presupuestaria que recogiese aquél. Y a tal fin se aprobó la anteriormente citada Orden de 20 de septiembre de 1989, que estableció la estructura, normas y códigos a los que debían adaptarse los Presupuestos de las Entidades Locales, siendo de aplicación tanto para éstas como para los Organismos autónomos de ellas dependientes 5 .

4 Esta Orden Ministerial resultó posteriormente modificada por la anteriormente citada Orden de 8 de septiembre de 1999 .

5 El citado Real Decreto 500/1990 de 20 de abril, desarrolló el Capítulo I del Título VI de la Ley 39/1988 en cuanto al contenido y aprobación de los presupuestos, los créditos y sus modificaciones, la ejecución y liquidación y normas específicas para Organismos autónomos y sociedad mercantiles. Se presenta así la citada norma reglamentaria como un auténtico «Reglamento Presupuestario» en el que, con excepción de la estructura de los presupuestos, se recoge una regulación completa de toda esta materia. Recuérdese que, con carácter general, es el Ministerio de Economía y Hacienda el encargado de establecer la normativa completa en materia contable de las Entidades Locales. Así se encargó de precisarlo en su día la Orden de 17 de julio de 1990 la cual, a su vez, aludía a otras dos disposiciones: la Instrucción de Contabilidad de la Administración Local, aprobada mediante dicha Orden y que sustituyó a la Instrucción de Contabilidad de 1952, conteniendo las normas contables generales así como la regulación de las operaciones contables, de los libros y documentos y de las cuentas y estados; y el Plan General de las Cuentas para las Entidades Locales, recogido como anexo a la misma instrucción. Tal y como establecía el art. 184 de la Ley 39/1988, esta Instrucción y el Plan General de Cuentas sólo resultaban aplicables a las Entidades Locales de 5.000 o más habitantes. Asimismo conviene hacer referencia a la anteriormente citada Orden 20 de septiembre de 1989, por la que se establece la estructura de los Presupuestos de las Entidades Locales; y a la Orden de 17 de julio de 1990, por la que se aprobó la Instrucción de Contabilidad para la Administración Local, actualmente sustituida por las Órdenes EHA/4040/2004, de 23 de noviembre, por la que se aprueba la Instrucción del modelo Básico de Contabilidad Local, 4041/2004, de 23 de noviembre, por la que se 
Téngase presente además que la Intervención General de la Administración del Estado (IGAE), en virtud de lo dispuesto en el art. 125.2 g) de la LGP, tiene encomendada la elaboración de las cuentas nacionales de las unidades que componen el sector de las Administraciones Públicas, dentro de las cuales se integran las Corporaciones Locales.

Al amparo de todo este marco normativo se configura un régimen presupuestario local sustancialmente idéntico al del Estado y orientado a proyectar sobre las Corporaciones Locales el conjunto de previsiones contenidas en la Ley General Presupuestaria y en el Real Decreto Legislativo 2/2007, de 28 de diciembre, por el que se aprueba el Texto Refundido de la Ley General de Estabilidad Presupuestaria. Ambos textos legales son de aplicación directa en las Entidades Locales, al margen de la competencia que en esta materia puedan tener atribuidas las Comunidades Autónomas.

Podemos concluir por tanto que la regulación presupuestaria de las Entidades Locales se encuentra acomodada, con las especificidades propias de la Administración Local, a la normativa presupuestaria del Estado prevista en Ley 47/2003. De hecho en numerosas ocasiones los textos del TRLHL y los de la LGP coinciden en su totalidad, viniendo a ocupar el RDLeg. 2/2004 en el ordenamiento jurídico local, y por lo que a dichas materias se refiere, un lugar análogo al que ocupa la LGP en la normativa estatal.

Debe reconocerse sin embargo que esta intención del legislador de homogeneizar, en cierta medida, la actividad presupuestaria de la Administración Central y la de las Entidades Locales no ha sido todo lo eficaz que debiera, ya que no existe un precepto concreto que armonice estas actuaciones cada vez que la Administración Central modifica su modus operandi, siendo necesario para el caso de las Entidades Locales una norma específica al respecto. En este sentido, a lo largo de la vigencia de estas disposiciones para el ámbito local se ha ido produciendo un cierto distanciamiento entre las dos Administraciones, ya que para la Administración General del Estado se origina cada año una modificación o adaptación a la circunstancias del momento, lo que no se produce en el ámbito de la Administración Local, con el consiguiente problema para la homogenización que ello implica.

\section{CONFIGURACIÓN DEL PRESUPUESTO POR PROGRAMAS: CONCEPTO Y OBJETIVOS}

Históricamente los antecedentes más remotos de la aplicación del sistema del presupuesto por programas los encontramos en la empresa privada, siendo General Motors, en 1924, la pionera en la utilización de esta técnica presupuestaria. Con posterioridad el presupuesto por programas comenzó a ser aplicado por la Administra-

86 aprueba la Instrucción del modelo Normal de Contabilidad Local y 4042/2004, de 23 de noviembre, por la que se aprueba la Instrucción del modelo Simplificado de Contabilidad Local. 
ción norteamericana. Fue concretamente el Departamento de Defensa norteamericano (siendo Secretario del mismo Robert Mcnamara) donde, en 1961, se utilizó por vez primera esta técnica. Años más tarde, hacia 1965, se extendió por orden del entonces presidente Lyndon B. Jhonson y bajo la denominación «Sistema de Planificación, Programación y Presupuestación» (P.P.B.S.), a toda la Administración Federal. Y ello con la finalidad de lograr la consecución de tres objetivos: hacer las cosas mejor, con mayor rapidez y con el menor coste.

Dentro de nuestro ámbito presupuestario la aplicación del sistema de presupuesto por programas tuvo su primer precedente en la antigua Ley General Presupuestaria de 1977, cuyo art. 52 disponía que en la estructura de los gastos han de contemplarse las finalidades u objetivos que con ellos se proponga conseguir, añadiendo a continuación su art. 53 que «Se aplicará una clasificación funcional desagregada por programas y los Entes con presupuesto diferenciado establecerán un sistema de objetivos que sirva de marco a su gestión presupuestaria y de conformidad con ellos, se clasificarán los créditos por programas». Ya en el año 1979 fue parcialmente aplicado este sistema de presupuesto, procediéndose en 1984 a elaborar los primeros presupuestos por programas.

Con carácter general el sistema del presupuesto por programas (PPBS) pretende alcanzar la eficacia de los centros gestores basando sus fines en la resolución de los problemas sociales y en la elección de la solución más satisfactoria. En líneas generales nos hallamos ante un proceso integrado de gestión, en el que se analiza la actividad administrativa en su totalidad, asignándose los recursos entre programas alternativos. Se caracteriza pues esta modalidad presupuestaria por ser la primera vez que se integra la presupuestación, la programación y la planificación económica, configurándose el presupuesto como la secuencia anual del plan concretado en programas.

A nuestro juicio el presupuesto por programas supone la integración de las dos visiones del presupuesto, a saber: la macroeconómica, como instrumento de política económica, y la microeconómica, como medio para la asignación de los recursos disponibles. Dicho presupuesto se centra en la planificación estratégica, consistiendo su propósito final en contribuir a la racionalización de las elecciones del Sector público mediante la utilización de técnicas de análisis económico. En este sentido presenta un carácter complejo, ya que no sólo exige una cuidadosa identificación de los objetivos perseguidos, sino además la elaboración de las diferentes alternativas existentes para alcanzarlos y una cuantificación económica de cada una de ellas.

¿Cabe aludir a la existencia de alguna diferencia entre este tipo de presupuesto y el presupuesto de ejecución o tareas? En nuestra opinión, así es. Piénsese que el presupuesto por funciones pretende seleccionar los mejores métodos para administrar un programa. En cambio, el presupuesto por programas persigue seleccionar los mejores programas. Por otra parte, mientras el presupuesto por funciones busca la mejora de la eficacia y de la eficiencia de la Administración al nivel de servicios concretos o del conjunto organizativo gestor de una función, el presupuesto por programas 
confecciona categorías de datos con la finalidad de poder comparar los diversos programas de los que se pueda disponer para conseguir un fin determinado. En definitiva, el presupuesto por funciones presenta un carácter particularista, mientras que el presupuesto por programas tiene una vocación de generalidad.

En todo caso a través de esta forma de presupuestación se permite conocer lo que ha hecho cada departamento ministerial, sus resultados (actividades) y el coste de su realización. Asimismo se unen los procesos de elección política y presupuestación, configurando el presupuesto como un instrumento para planificar la política y mejorar la asignación de los recursos.

Debe precisarse no obstante que no cabe aludir a la existencia de un modelo único, dado que se ve afectado por las características políticas y administrativas de cada país. De este modo el Presupuesto por programas se desliga de la concepción tradicional del concepto presupuestario. La adopción de este tipo de presupuestación supone la aceptación de unos elementos fundamentales que pasamos a analizar.

En primer lugar, los objetivos de gasto. Estos objetivos no son sino el resultado que se espera obtener durante un período de tiempo determinado a través de la aplicación de los recursos asignados. Téngase presente que cada programa representa la ordenación de aquellos medios de los que disponen los centros gestores en orden a la consecución de sus objetivos.

Con carácter general, las distintas etapas que han de seguirse para la fijación de los objetivos pueden concretarse en las siguientes: selección del objetivo, analizando las necesidades; discusión del objetivo seleccionado, procediéndose a delimitar los objetivos seleccionados; y fijación del objetivo, consistente en determinar los objetivos a alcanzar y establecer su cuantificación Una vez fijados estos objetivos, será necesario establecer una estructura organizada en la que exista un responsable y una unidad administrativa, con la finalidad de conseguir los objetivos establecidos.

En segundo término hemos de referirnos a los programas, que representan combinaciones de medios identificables orientados al logro de un determinado objetivo a alcanzar por un centro de gestión. El programa constituye por tanto el conjunto de actividades mediante las cuales se pretenden conseguir los objetivos propuestos en un determinado plazo de tiempo. A través del mismo se definen claramente las actividades que deben desarrollarse, así como el centro gestor que ha de llevarlas a cabo, los objetivos que se desean conseguir y los indicadores que faciliten la información acerca de la consecución o no de los objetivos, de la realización de los programas y de utilización de los recursos.

La elaboración de los programas se puede realizar desde dos perspectivas: de una parte, estableciendo los objetivos y elaborando los programas en base a aquéIlos; y, de otra, elaborando los programas en base a la estructura administrativa, agru88 pando las actividades y fijando los objetivos. Existen además diversas clases de pro- 
gramas: finalistas, de apoyo, de responsabilidad y de responsabilidad compartida o de intervención.

La evaluación de todo programa consiste en el examen de la ejecución del mismo, al objeto de determinar los resultados conseguidos respecto de los objetivos propuestos. Dicha evaluación comprende operaciones tales como las de: análisis de los costes y de los beneficios obtenidos; examen comparativo de los objetivos proyectados y de los realmente obtenidos al objeto de hallar las causas de la desviación en estos resultados; análisis de los motivos o causas de las desviaciones acontecidas entre lo conseguido y lo proyectado; y revisión de todas las decisiones adoptadas, reestructurando los programas, revisando los objetivos y reasignando los recursos tanto humanos como materiales y financieros.

Por su parte los indicadores constituyen elementos referidos a los objetivos de los programas presupuestarios que proporcionan informaciones cuantificables, presentando un carácter descriptivo y valorativo.

Finalmente, en todo sistema de Presupuesto por programas resulta imprescindible el establecimiento de algún tipo de control referido a la ejecución de los programas y sus resultados finales. En otras palabras, ha de llevarse a cabo un seguimiento, control y evaluación de los resultados de la gestión.

\section{PRINCIPIOS INFORMADORES DEL PRESUPUESTO POR PROGRAMAS}

¿Cuáles son los principios básicos que informan el presupuesto por programas? En primer lugar hemos de aludir al llamado principio de racionalidad, de conformidad con el cual la programación de los presupuestos debe realizarse de una manera científica a través del razonamiento sistemático. En efecto, las posibilidades de actuación deben ser valoradas, tanto en sus ventajas como en sus inconvenientes, al objeto de tomar aquella decisión más coherente que posibilite el aprovechamiento máximo de los recursos.

De acuerdo con el principio de previsión, la programación constituye una previsión de actuaciones a realizar en el futuro. El necesario respeto al principio de universalidad demanda que los programas abarquen todos los sectores de la Administración, así como todas las fases del proceso económico, social y administrativo. Y, en aras de satisfacer el principio de unidad, los diferentes planes deben estar integrados entre sí, formando un cuerpo único y compatible.

Al amparo del principio de continuidad los planes deben tener vocación de continuidad de manera que, cumplido un plan, se formule el siguiente o se revise el concluido, permaneciendo la secuencia de la actividad emprendida. Finalmente, la aplicación del principio de inherencia resulta necesaria en cualquier organización humana, siendo igualmente inherente a la propia Administración. 


\section{PRINCIPALES FASES DEL PRESUPUESTO POR PROGRAMAS}

Sin lugar a dudas, el Presupuesto por programas constituye un instrumento indispensable para la mejora de las fases del ciclo presupuestario. En la elaboración, mediante la preparación por el responsable de cada programa de soluciones alternativas en programas plurianuales para cada campo de actividad. En la aprobación, presentando la actividad del Estado al Parlamento y a los ciudadanos, de modo que puedan conocerse los metas u objetivos a alcanzar y los medios asignados para su consecución.

En la ejecución, responsabilizando a los gestores de la consecución de los objetivos programados, medibles a través del establecimiento de indicadores de resultados. $Y$ en el control, evaluando su nivel de eficacia y de eficiencia y efectuando las oportunas correcciones de las desviaciones observadas.

El proceso presupuestario por programas se desarrolla siguiendo su ciclo, a través de las siguientes fases sucesivas e integradas. Las tres primeras previas a su ejecución y la última simultánea o posterior: 1. Planificación. 2. Programación. 3. Presupuestación. 4. Ejecución. 5. a Control.

La planificación persigue la búsqueda de un futuro deseado y con tal fin, previo análisis de las alternativas posibles, establece los medios precisos para conseguirlo. A través de la planificación se realiza el estudio de las nuevas actuaciones a emprender, así como de las que ya han sido puestas en marcha anteriormente, con la finalidad de concretar la mejor política a seguir al objeto de satisfacer las necesidades sociales.

El proceso planificador debe ser por tanto integral, incluyendo a toda la organización aunque pueda desarrollarse en un ámbito temporal o material diferente. Los resultados alcanzados y la información obtenida deben servir para mejorar los futuros planes y rectificar en su caso los objetivos, estrategias o acciones.

La programación, que es la principal fase, tiene por objeto el establecimiento de los programas de gasto a ejecutar. Una vez realizada la planificación y concretados los objetivos a cumplir, se produce la agrupación de éstos por programas, lo que determina la estructura funcional del presupuesto para un determinado período de tiempo. Así las cosas, una vez determinados los programas se asignan los recursos presupuestarios precisos para financiar durante el correspondiente ejercicio presupuestario los medios personales, materiales y financieros necesarios para alcanzar los objetivos programados en dicho período. La presupuestación representa por tanto aquella fase mediante la cual se asignan todos los recursos necesarios (tanto personales como materiales y financieros) para la consecución de los objetivos recogidos en los programas.

Estas tres primeras fases se distinguen por su duración: a largo plazo; a medio plazo y anual, respectivamente. Asimismo se diferencian por el grado de concreción 90 y duración de sus objetivos: generales o políticos; sectoriales y específicos. 
Queda claro por tanto que los programas deben recoger los créditos que se han de destinar para alcanzar los objetivos, así como los indicadores que permitan evaluar hasta qué punto se han conseguido dichos objetivos.

Con posterioridad, durante su vigencia, se procederá a gestionar el presupuesto (ejecución) aprobado, con el fin de alcanzar a través de los medios asignados las metas u objetivos previstos. La ejecución implica además el control de la gestión, que se efectúa analizando los resultados obtenidos así como las desviaciones acaecidas entre lo previsto y lo conseguido, con el objetivo de descubrir las causas que las han ocasionado.

El ciclo se cierra con un control y evaluación de las metas u objetivos alcanzados, que sirve para la retroalimentación del sistema, ayudando en base a lo conseguido y a sus circunstancias de ejecución a asignar los recursos en los ejercicios futuros.

Recapitulando, el presupuesto por programas representa una técnica destinada a asignar los recursos entre fines competitivos. En nuestro país la clasificación de los créditos por programas se planteó en su día con la antigua Ley General Presupuestaria de 4 de enero de 1977. Se inició así un proceso de varios años que culminó con el primer presupuesto por programas, introducido en la Ley de Presupuestos Generales del Estado para el año 1989, y que vino a definir una serie de programas y los procedimientos de seguimiento del grado de realización de los objetivos definidos en las memorias de cada programa.

Actualmente la Ley 47/2003 plantea la relación entre la programación presupuestaria y el objetivo de estabilidad. En concreto, la programación de la actividad del Sector público estatal se materializa en los escenarios presupuestarios plurianuales, tanto de ingresos como de gastos, que deben ajustarse al objetivo de estabilidad para los tres ejercicios siguientes y a los que se adecuarán los Presupuestos Generales del Estado. Asimismo se definen los programas plurianuales ministeriales y de los Centros Gestores y se vincula la asignación de recursos de forma prioritaria con el nivel de cumplimiento de los objetivos en ejercicios anteriores, como de forma específica recoge su art. 31.5, de conformidad con el cual.

\section{LA PROYECCIÓN DEL PRESUPUESTO POR PROGRAMAS EN EL MARCO DE LAS HACIENDAS LOCALES}

\section{Introducción}

Como entes sujetos a régimen presupuestario que son, las Haciendas Locales han de someter su actividad económico-financiera dentro de los límites contenidos en su propio presupuesto en términos de gastos y de recursos necesarios para su fi- 
nanciación, constituyéndose así el documento presupuestario como un instrumento principal y vertebrador de su gestión ${ }^{6}$.

Las obligaciones que, como máximo, pueden reconocer la Entidad y sus Organismos autónomos, así como los derechos que prevean liquidar durante el correspondiente ejercicio, están haciendo referencia a la existencia de unos gastos (los cuales presentan un carácter limitativo que garantiza que los importes de las partidas del presupuesto de gastos no vayan a ser sobrepasadas) y de unos ingresos, ya que el presupuesto constituye una previsión sobre la base de la cual han de planificarse los gastos que puedan ser realizados en el ejercicio presupuestario. Los ingresos señalados en el presupuesto son pues fundamento y justificación de los gastos?.

En cuanto a las previsiones de ingresos y gastos de las Sociedades Mercantiles cuyo capital social pertenezca íntegramente a la Entidad Local correspondiente, dichas previsiones presentan, salvo que el Pleno de la Corporación disponga un régimen especial, un carácter meramente estimativo, adoleciendo así de un carácter limitativo. Piénsese además que, a pesar de que la terminología comúnmente utilizada habla de gastos e ingresos, lo que realmente contiene un presupuesto son obligaciones y derechos, concepto jurídico que abarca un campo sensiblemente más amplio. Así, por ejemplo, el mal llamado «presupuesto de gastos» incluye conceptos tales como inversiones, concesión de préstamos o amortización de deuda; y, por su parte, el "presupuesto de ingresos» contempla también entradas de fondos provenientes de operaciones crediticias pasivas o reintegros de préstamos concedidos.

Cabe hablar, por tanto, de gasto e ingreso presupuestario versus gasto e ingreso económico. Es esta, además, una singularidad que se refleja en la normativa contable a través del llamado Plan General de Contabilidad Pública de 1994 (PGCP) y, más precisamente, en su glosario de términos. Señala concretamente el citado Plan en relación con los gastos que mientras los gastos presupuestarios son aquellos flujos que suponen el empleo de créditos consignados en el presupuesto de gastos de la entidad (conllevando su realización obligaciones a pagar presupuestarias con origen en gastos, en inversiones o en el vencimiento de obligaciones y constituyéndose como el componente negativo del estado resultado presupuestario de la entidad), los gastos (económicos) son aquellos flujos que configuran el componente negativo del estado resultado económico-patrimonial, producidos a lo largo del ejercicio por las operaciones conocidas de naturaleza presupuestaria o no presupuestaria, como consecuencia de la variación de activos o el surgimiento de obligaciones, y que implican un decremento en los fondos propios.

6 Véase a este respecto Esteso Ruiz, P. y Melguizo Sánchez, A., (1986) «Haciendas locales y Presupuestos del Estado", Hacienda Pública Española, núm. 103, pp.. 172 y ss. y, más recientemente, Iglesias Alonso, A., (2007) «El presupuesto como instrumento de gobernanza e innovación en la gestión pública local: estudio de caso», Tributos Locales, núm. 71, pp.. 19 y 20.

7 Ello justifica, además, que se disponga la obligación de presentar, junto con el presupuesto de la Entidad municipal, un informe económico-financiero. 
Nótese por tanto que este último término se reserva para aquellos flujos que deben imputarse al resultado citado de la Entidad a través de cuentas de gestión, lo que explica que no deban confundirse los términos gasto y gasto presupuestario. De hecho existen gastos que no constituyen gasto presupuestario (por ejemplo, las dotaciones a las provisiones y a la amortización del inmovilizado); e igualmente existen gastos presupuestarios que no constituyen gasto (por ejemplo, la inversiones o la concesión de préstamos). Tampoco ha de confundirse con el término pago, ya que un gasto no debe identificarse con flujos de tesorería.

Refiriéndose a los ingresos señala el PGCP que mientras los ingresos presupuestarios constituyen aquellos flujos que determinan recursos para financiar los gastos presupuestarios de la Entidad, generando derechos de cobro presupuestarios y debiendo imputarse al correspondiente Presupuesto, con origen en ingresos, en otros recursos o en el vencimiento de derechos de cobro no presupuestarios (lo que posibilita su constitución como el componente positivo del estado resultado presupuestario de la entidad), los ingresos (económicos) se definen como aquellos flujos que configuran el componente positivo del estado resultado económico-patrimonial, producidos a lo largo del ejercicio por las operaciones conocidas de naturaleza presupuestaria o no presupuestaria como consecuencia del incremento de los activos o de la disminución de las obligaciones que implican un incremento del patrimonio neto.

Por tanto el citado término se reserva para aquellos flujos que deben imputarse al resultado de la Entidad a través de cuentas de gestión. Quiere decirse con ello que no deben confundirse los términos ingreso e ingreso presupuestario. Así, existen ingresos que no constituyen ingreso presupuestario (por ejemplo, las provisiones aplicadas a su finalidad o los trabajos realizados por la empresa para su inmovilizado); y existen ingresos presupuestarios que no constituyen ingreso (caso de la enajenación de inversiones o del reintegro de préstamos concedidos). Tampoco debe confundirse con el término cobro, es decir, un ingreso no debe identificarse con flujos de tesorería.

Señala a este respecto Dodero Jordán ${ }^{8}$ que «La premisa de que se contraiga una obligación es inherente al gasto presupuestario y no al gasto económico; así, las dotaciones a las provisiones y a la amortización del inmovilizado, al no suponer una obligación para la entidad, carecen de significado desde el punto de vista presupuestario».

Carecen igualmente de la característica esencial de ser un derecho de cobro algunos ingresos que, en cambio, sí lo son de naturaleza económica; este es el caso de las provisiones aplicadas a su finalidad o de los trabajos realizados por la empresa para su inmovilizado. En ambos casos quedan privados de la característica esencial de ser un derecho de cobro.

8 Dodero Jordán, A., (2002), Teoría y práctica de la contabilidad presupuestaria en el Sector Público (Especial referencia a las entidades locales), Estudios de Administración Local, Comares, Granada, p. 271. 
En definitiva, son los gastos e ingresos económicos los que integran la cuenta de resultado económico-patrimonial y los gastos e ingresos presupuestarios quienes generan el nacimiento de una obligación de pago o de un derecho de cobro presupuestario, respectivamente, permitiendo al gestor al finalizar el ejercicio configurar, entre otras magnitudes de carácter presupuestario básicas para la toma de decisiones, el resultado presupuestario.

A pesar de ello, siempre que se habla de obligación de pago y de derecho de cobro en términos generales no cabe ceñirse estrictamente al ámbito presupuestario ya que, en el seno de una Entidad Pública, también se generan obligaciones y derechos de naturaleza no presupuestaria; las primeras nacen como consecuencia de la existencia de un Presupuesto, mientras que las segundas reflejan operaciones de contenido patrimonial, si bien al margen del Presupuesto.

Pues bien, es en este marco donde se establece una nueva estructura presupuestaria que responde al esquema existente para la Administración del Estado, consagrándose el «Presupuesto por programas». Tal y como señala el art. 167 del TRLR$\mathrm{HL}$, el Ministerio de Economía y Hacienda establecerá con carácter general la estructura de los Presupuestos de las Entidades locales teniendo en cuenta la naturaleza económica de los ingresos y de los gastos, así como las finalidades u objetivos que con estos últimos se propongan conseguir, recomendándose, por lo tanto, la formación del Presupuesto por programas en el que se fijen objetivos y fines a alcanzar, y no su elaboración en base a un simple incremento de gastos respecto al presupuesto del ejercicio anterior, característica intrínseca al presupuesto tradicional, de medios o administrativo.

Se presenta así una nueva estructura presupuestaria con las clasificaciones orgánica, funcional y económica del gasto. $Y$ es que, si bien con anterioridad a la promulgación de la extinta LRHL se anteponía la clasificación económica a la funcional (lo cual imposibilitaba o dificultaba en gran medida la elaboración de presupuestos por programas), la actual normativa se caracteriza por anteponer la funcional a la económica, presentándose por tanto bajo una clasificación funcional económica. Las Corporaciones podrán así establecer la clasificación orgánica atendiendo a su propia estructura de acuerdo con sus reglamentos o decretos de organización.

Con carácter general los Presupuestos de las Entidades Locales han de estructurarse desde una perspectiva funcional, económica y/u orgánica. Ahora bien, mientras que el primero de estos criterios resulta aplicable, de acuerdo con la legislación vigente, únicamente al presupuesto de gastos, su clasificación orgánica es optativa, aunque ya son muchas las Administraciones Locales que la aplican.

Como seguramente se recordará, ya en su día el art. 148 de la antigua Ley 39/1988 se encargó de precisar que los estados de gastos de los Presupuestos Generales de las Entidades Locales han de aplicar: la clasificación funcional, en la que ha de quedar 94 integrada, en su caso, la clasificación por programas (que consta de tres niveles: gru- 
po de función, función y subfunción, pudiendo ampliarse en uno o dos niveles); y la clasificación económica (que presenta separadamente los gastos corrientes y los de capital, constando igualmente de tres niveles, capítulo, artículo y concepto, y pudiendo ampliarse en uno o dos niveles) ${ }^{9}$.

Centrando nuestro análisis en aquello que establece la vigente normativa presupuestaria, si bien es cierto que el Texto Refundido de la Ley Reguladora de las Haciendas Locales recomienda la formación de un presupuesto por programas en el que se fijen objetivos y fines a alcanzar, se encarga a continuación de clasificar funcionalmente los créditos para gastos por grupos de función, funciones y subfunciones, admitiéndose así que aquellas Entidades que lo consideren conveniente para una mejor gestión del mismo puedan descender en la clasificación funcional al nivel de programa e incluso subprograma.

Nótese por tanto que la elaboración de programas de gasto no es, en principio, obligatoria, sino voluntaria, si bien de forma indirecta se está obligando a un determinado conjunto de Entidades Locales a confeccionar su presupuesto por programas.

\section{Criterios clasificatorios de los presupuestos de los entes locales}

Tal y como tuvo oportunidad de establecer la Orden de 20 de septiembre de 1989, reguladora de la estructura de los presupuestos de las Entidades Locales, estos Entes han de elaborar sus presupuestos teniendo en cuenta la naturaleza económica de los ingresos y de los gastos, y las finalidades u objetivos que con estos últimos se propongan conseguir, pudiendo clasificar los gastos e ingresos atendiendo a su propia estructura organizativa.

Así las cosas estas Entidades han de presentar obligatoriamente los gastos presupuestarios según su naturaleza (clasificación económica) y según la finalidad u objetivos que se pretender conseguir (clasificación funcional), anteponiéndose la funcional a la económica y siendo optativa su presentación en base al órgano gestor del gasto (clasificación orgánica).

En lo relativo a los ingresos presupuestarios, éstos han de estructurarse según su naturaleza (clasificación económica), no existiendo una posible estructura funcional ya que los ingresos de mayor volumen, procedentes de los impuestos directos (Impuesto sobre Bienes Inmuebles e Impuesto sobre Actividades Económicas) y de participaciones en los tributos del Estado tienen carácter no finalista y, por ello, no

9 En todo caso los niveles de capítulo y artículo han de ser los mismos que los establecidos para la Administración del Estado. Véase a este respecto López Hernández, A. M. y Buendía Carrillo, D., (1993), «Presupuesto de objetivos y Contabilidad Analítica en las entidades locales», I Congreso Nacional de la Asociación Española de Contabilidad Directiva, Madrid,, p. 62 y Pou Díaz, J. y Sanz Monge, E., (1993) «La nueva normativa presupuestaria y contable en la Administración Local», Partida Doble, núm. 6, pp. 48 y 49. 
son asignables a gastos específicos. $\mathrm{Y}$, por lo que respecta a su configuración bajo una estructura orgánica, su aplicación en las Entidades Locales carecería de sentido ya que, generalmente, la gran mayoría de ingresos se gestionan a través del área económico-financiera, de acuerdo con el principio de caja única.

Ciertamente, uno de los principales problemas existentes a la hora de elaborar un presupuesto está en delimitar los créditos del presupuesto de gastos a fin de que se ajusten lo más posible al importe total de las necesidades de la Entidad, y, con ello, se logren plenamente los objetivos y fines para cuyo cumplimiento se dotaron los correspondientes créditos.

Hasta 1988, año en el que, como se señaló con anterioridad, se promulgó la extinta LRHL, la fijación de este importe se llevaba a cabo en base a la técnica presupuestaria denominada presupuesto tradicional, de medios o administrativo, consistente en configurar el presupuesto del ejercicio como consecuencia de aplicar al del ejercicio anterior un coeficiente de incremento general, constituyendo el interés de esta técnica el establecimiento del control de legalidad de las actuaciones realizadas por la Entidad.

Con posterioridad, a partir de la aprobación de la citada LRHL y de los desarrollos normativos inmediatamente posteriores, se diseñó un marco legal que auspiciaba la transición de un Presupuesto de medios o tradicional a un Presupuesto de fines y objetivos. Tal y como ha señalado Barrio ${ }^{10}$, desde el punto de vista presupuestario los elementos más destacados de este marco legal son los siguientes. En primer lugar, se introduce la clasificación funcional en el Presupuesto, lo que permite la fijación de los objetivos que se persiguen con el gasto público y la definición de los programas presupuestarios diseñados para su consecución. Y, en segundo término, se modernizan los sistemas de control introduciendo, junto al tradicional de legalidad, el seguimiento y evaluación de los objetivos definidos y del coste de la prestación de servicios en base a criterios de eficiencia y eficacia.

De este modo surge el presupuesto por programas y objetivos como modelo presupuestario idóneo para su aplicación a la Administración Local, a pesar de que su implantación, en principio, no resulta obligatoria, sino más bien recomendable, obligando a la Corporación a fijar la actuación en el ejercicio que se presupuesta, independientemente de los gastos del ejercicio anterior, y dando una visión de los fines y objetivos a alcanzar, lo que permite incidir en los aspectos de gestión de los recursos otorgados a los responsables del ente. En relación a esta última figura, y como bien ha puesto de manifiesto Bujidos ${ }^{11}$, la estructuración de las Entidades Locales, como el caso de los Ayuntamientos, en concejalías, «deja perfectamente resuelta la determina-

10 Barrio, J., (2000), «La oficina presupuestaria: una necesidad en los entes locales», Análisis Local, núm. extraordinario, 2, p. 156.

96 p. 174.

11 Bujidos, P. (2000), «La experiencia de los ayuntamientos», Análisis Local, núm. extraordinario 2, 
ción de los responsables, pero el problema se complica cuando los objetivos son realizables por distintas concejalías».

Surge en consecuencia la obligación de establecer una adecuada distinción entre los objetivos asignados a cada programa, por una parte, y las dotaciones necesarias a las actividades de cada centro gestor responsable, por otra; a modo de síntesis, el programa puede tener varios objetivos y cada objetivo distintos centros responsables. Y es que, dado un sector de la complejidad del Sector público, la estructuración de actividades y objetivos es condición sine qua non para conseguir una racionalización de los procesos y de la gestión. En este sentido el desarrollo de una estructura presupuestaria, basada en programas coordinados, puede coadyuvar a mejorar la eficiencia del gasto y la eficacia en el cumplimiento de objetivos.

Pues bien, al objeto de poder integrar el conjunto de procesos de la Administración, el presupuesto por programas aporta una estructura general en la que quedan coordinadas las diversas funciones que la conforman, así como sus respectivos programas y objetivos. El diseño de estas grandes funciones muestra la distribución de los recursos públicos en necesidades de carácter general, educativo, cultural y social. De este modo el punto de referencia para la elaboración de esta técnica presupuestaria lo constituyen los objetivos de la actividad del Sector público, definiendo cada unidad de decisión para cada programa o subprograma concreto que le haya sido asignado los objetivos a alcanzar a lo largo del período presupuestario, ya que los objetivos a medio o largo plazo deben fijarlos las instancias políticas. Y ello a pesar de que los objetivos deberían definirse en términos de satisfacción de necesidades públicas y los programas diseñarse para lograr estos objetivos, cuestión que no siempre es sencilla de realizar.

A priori el tránsito de un presupuesto tradicional a otro concebido como instrumento de gobierno consiste, básicamente, en convertir al Presupuesto en el centro de debate de los problemas que la comunidad tiene planteados y de las alternativas y prioridades que el gobierno local propone para solucionarlos, a través de la asignación de los recursos públicos a los diversos programas de gasto. Quiere decirse con ello que no basta únicamente con definir los programas, siendo necesario además dotarlos de objetivos y fines.

El Presupuesto planificado por programas establece pues una clasificación general de categorías de gastos destinada a comparar los programas alternativos para alcanzar un objetivo determinado, pretendiendo de esta forma cuestionar la adecuación de cada programa presupuestario. En él se emplean, básicamente, tres técnicas destinadas a analizar las alternativas para realizar un programa presupuestario: análisis coste-beneficio, análisis coste-eficacia y análisis de sistemas.

Contrasta esta modalidad presupuestaria con el llamado Presupuesto Base Cero, surgido de la esfera del ámbito empresarial, y cuya esencia queda recogida en la siguiente reflexión: se parte de cero de los programas de gasto existentes, los cuales no tienen garantizada su permanencia, con el objetivo de reducir gastos superfluos o 
innecesarios. A título de ejemplo, los programas de Igualdad y Empleo pueden tratarse dentro de los objetivos fijados en las áreas de Seguridad, Empleo y la propiamente Social si, por razones orgánicas, se establecen concejalías distintas.

Surge no obstante la necesidad de llevar a cabo su seguimiento mediante la implementación de sistemas de control que permitan el análisis de las desviaciones en medios y objetivos y la puesta en marcha de los procesos dirigidos a la corrección de dichas desviaciones, es decir, hacia una mayor eficiencia y eficacia en la gestión del gasto local. En esta línea se sitúa, además, la necesidad de que los gestores públicos cuenten con instrumentos de control que les permitan un seguimiento de los programas, detectando las desviaciones entre previsiones y resultados, facilitando el análisis de sus causas y posibilitando con ello la toma de decisiones. Obviamente, aquellas Entidades que utilizan el presupuesto por programas o una aproximación de los mismos valorarán más la utilidad del presupuesto como instrumento de planificación y control que aquéllas que únicamente utilizan el presupuesto tradicional, fomentando la introducción de criterios de eficiencia y eficacia en la asignación de recursos.

Lo cierto es que en la actualidad la sociedad española demanda al Estado, a las Comunidades Autónomas y a las Corporaciones Locales (éstas últimas, además, como Administración más cercana al ciudadano que son), más y mejores servicios y, en especial, que la seguridad ciudadana, el sistema de educación y la sanidad funcionen correctamente, cuidándose el medio ambiente y mejorándose las infraestructuras. Y, de cara a dar cumplimiento a dichas demandas, cabe aludir a la existencia de un primer plano estrictamente político de fijación de objetivos y prioridades, así como de un segundo plano de gestión que exige rigor, control y eficacia en el logro de los objetivos que persiguen quienes han sido democráticamente elegidos por la sociedad para ejercer el gobierno de las Administraciones Públicas.

Esta orientación hacia el ciudadano, su cliente, a la hora de ejercer sus competencias, esto es, prestar sus servicios, es una de las características más importantes de la nueva gestión pública o gerencialismo, e implica conocer las necesidades de los ciudadanos y diseñar una estrategia para atenderla que tome en consideración los servicios que aquéllos esperan y requieren de la Entidad y la forma más eficaz, eficiente y económica de atenderlos. En palabras de Montesinos Julvé12, "La nueva gestión pública supone un reto para la institución presupuestaria, que ha de permitir una asignación adecuada de los recursos económicos, desplazando su énfasis hacia los objetivos y otorgando un amplio grado de flexibilidad en la gestión de los responsables».

En suma, para poder sentar las bases de una nueva cultura de gestión en la Administración Local el Presupuesto se ha de convertir, paulatinamente, en un esfuerzo integrador de la voluntad política y de la capacidad técnica destinado a rentabilizar los recursos asignados, definir prioridades y objetivos, fijar criterios de evaluación,

12 Montesinos Julve, V. (1999), «El cambio de la cultura organizativa de las Administraciones Públicas y el presupuesto», Análisis Local, núm. extraordinario 2, p. 73. 
adecuar los recursos humanos y materiales, mejorar y evaluar la calidad de los servicios prestados y modernizar la Administración.

Ahora bien, a pesar de que el anteriormente citado art. 167 del TRLHL recomienda la formación de un presupuesto por programas en el que se fijen objetivos y fines a alcanzar, seguidamente clasifica funcionalmente los créditos para gastos por grupos de función, funciones y subfunciones, admitiéndose que aquellas Entidades que lo consideren conveniente para una mejor gestión del mismo puedan descender en la clasificación funcional al nivel de programa e incluso subprograma. Esto conlleva que, para un Ente Local, el detalle de los créditos deba presentarse, al menos, a nivel de subfunción; en consecuencia, la elaboración de programas de gasto no es, en principio, obligatoria, sino voluntaria.

Como seguramente se recordará, la Orden Ministerial de 20 de septiembre de 1989 presentaba una clasificación funcional del presupuesto de gastos dentro de cuyos Grupos de función figuraban los siguientes gastos imputables. En primer lugar, los Ilamados servicios de carácter general, es decir, gastos relativos a actividades que afectan, con carácter general, a todo el ente, y que consisten en el ejercicio de funciones de gobierno o de apoyo administrativo a toda la organización. Dentro de este Epígrafe quedan recogidos aquellos gastos generales que no puedan ser imputados directamente a ningún otro grupo de función.

En segundo término, el Epígrafe relativo a la protección civil y la seguridad ciudadana, que comprende aquellos gastos originados por los servicios que tienen a su cargo el orden y la seguridad, tales como policía municipal, protección civil, bomberos, etc. El tercer Epígrafe sería el relativo a seguridad, protección y promoción social, esto es, gastos y transferencias que constituyen el régimen de previsión y seguridad social a cargo de la Entidad, englobando: pensiones de funcionarios; atenciones de carácter benéfico-asistencial; atenciones a grupos con necesidades especiales, como jóvenes, discapacitados físicos y tercera edad, etc.

Dentro del Epígrafe cuarto, relativo a la producción de bienes públicos de carácter social, quedarían incluidos aquellos gastos efectuados en relación con la sanidad, educación, vivienda y urbanismo y, en general, todos aquellos tendentes a la elevación o mejora de la calidad de vida.

El Epígrafe quinto, bajo el título «Producción de bienes públicos de carácter económico», comprendería los gastos, preferentemente de inversión, relacionados con actividades que tienden a desarrollar el potencial económico del entorno. Este sería el caso de las infraestructuras básicas y de transportes, comunicaciones, infraestructuras agrarias, investigación científica y técnica, información básica y estadística, y otros de naturaleza análoga13.

13 Igualmente habría que incluir dentro del mismo las transferencias a otros agentes que colaboren en el cumplimiento de estos fines. 
Los Epígrafes sexto y séptimo, relativos a la regulación económica de carácter general y a la regulación económica de sectores productivos, comprenderían los gastos de los servicios relacionados con asuntos económicos, financieros y comerciales y los gastos derivados de actividades, servicios y transferencias que tienden a desarrollar el potencial económico de los distintos sectores de la producción, como agricultura, ganadería y pesca, industria, energía, minería y turismo, respectivamente.

Finalmente dentro del Epígrafe 9, bajo el título «Transferencias a otras Administraciones Públicas», habría que incluir las transferencias de carácter general que no pueden ser aplicadas a ningún otro Epígrafe de esta clasificación funcional, ya que en caso contrario se imputarían en la función específica correspondiente. Y, por lo que respecta al Epígrafe 0 , referente a la Deuda pública, comprendería los gastos de intereses y amortización de la deuda y demás operaciones financieras de naturaleza análoga, con exclusión de aquellos gastos que ocasione la formalización de las mismas.

Por otra parte, si acudimos a la normativa reguladora el art. 211 del TRLHL, pone de manifiesto que aquellos municipios de más de 50.000 habitantes y las demás Entidades Locales de ámbito superior, cuando confeccionen la Cuenta General de cada ejercicio, deberán realizar las siguientes memorias: una memoria justificativa del coste y rendimiento de los servicios públicos; y una memoria demostrativa del grado en que se hayan cumplido los objetivos programados con indicación de los previstos y alcanzados, con su coste.

Ahora bien, el principal problema radica en que, ni en el citado RDLeg. 2/2004 ni en el Real Decreto 500/1990 por el que se desarrollaba la antigua Ley Reguladora de las Haciendas Locales en materia presupuestaria, se indica explícitamente que las Entidades Locales deban elaborar un presupuesto por objetivos, por lo que difícilmente podría exigirse la elaboración de una memoria cuya base es algo que no existe. Podría considerarse, eso si, que dentro del concepto de programa al que se refiere el cuarto nivel de la clasificación del presupuesto en vigor (establecida como ya sabemos por la Orden Ministerial de 20 de septiembre de 1989) se entiende de forma implícita que deben contenerse objetivos; pero, aún en este caso, conviene recordar que el desglose a nivel de programa se considera como optativo en la citada Orden, por lo que la Entidad Local puede perfectamente confeccionar su presupuesto a nivel de subfunción, eludiendo de esta forma la discusión sobre si los programas han o no de incorporar objetivos. Lo que sí parece claro es que la única forma de poder realizar la citada memoria ex post es el haber programado los objetivos ex ante. $\mathrm{Y}$, además, difícilmente podrá indicarse cuáles de los objetivos previstos se han alcanzado si no se han establecido previamente los criterios de medición o indicadores, que supondrían algo así como las reglas del juego a las que habrá de atenerse la Memoria. En consecuencia, si se pretende ofrecer a los ciudadanos la discutida memoria de grado de cumplimiento de los objetivos habrá que empezar por fijarlos previamente.

En la línea de lo expuesto en el TRLHL, la Ley 57/2003, de 16 de diciembre, de 100 Medidas para la Modernización del Gobierno Local, en su art. 133, establece que la 
gestión económico-financiera de las Entidades Locales se ajustará, entre otros, a los siguientes criterios: la introducción de la exigencia del seguimiento de los costes de los servicios (apartado e); y la asignación de recursos, con arreglo a los principios de eficacia y eficiencia, realizada en función de la definición y el cumplimiento de objetivos (apartado f).

Ya la propia Ley 47/2003, General Presupuestaria, aprobada con anterioridad, se encargó de poner el énfasis en la eficiencia en la asignación de recursos, estableciendo la definición de objetivos como condición para la asignación de créditos presupuestarios, e introduciendo la normativa básica para iniciar procedimientos de evaluación de políticas públicas como instrumento de análisis de la efectividad de los recursos presupuestarios asignados a las mismas.

Téngase presente además la aplicación de Orden de 23 de noviembre de 2004, del Ministerio de Economía y Hacienda, por la que se aprueba, entre otras, la Instrucción del Modelo Normal de Contabilidad Local, norma de aplicación obligatoria para aquellas Entidades Locales cuyo presupuesto exceda de 3.000.000 euros, así como para aquellas otras cuyo presupuesto no supere este importe pero exceda de 300.000 euros y cuya población supere los 5.000 habitantes; e igualmente a las demás Entidades Locales, siempre que su presupuesto exceda de 3.000.000 euros, y a los Organismos autónomos dependientes de las Entidades anteriores. En la citada Orden y, más concretamente, en el apartado destinado a la formación de la Cuenta General, se hace referencia, con el literal exacto, a lo contenido en el art. 211 del TRLHL.

Puede concluirse así que, al menos de forma indirecta, se está obligando a un determinado conjunto de Entidades Locales a confeccionar su presupuesto por programas. 
